\title{
IS AMBLYOPIA AN IMPEDIMENT TO BINOCULAR FUNCTION?
}

\author{
ROBERT F. HESS \\ Montreal, Quebec, Canada
}

\begin{abstract}
SUMMARY
While there is good agreement that the strabismic and/ or anisometropic deficit in early life plays a key role in disrupting developing binocular connections, it is still not clear what impediment amblyopia is to binocular function in the adult. The answer to this depends upon our combined understanding of the normal stereo mechanism and the amblyopic deficit.
\end{abstract}

Strabismus/anisometropia, amblyopia and impaired binocular vision are part of the syndrome that we refer to as strabismic/anisometropic amblyopia. While our knowledge of each of these has improved over the last two decades, it is still not clear how exactly they interrelate. Is amblyopia the cause or the consequence of the breakdown in binocular function? The degree to which amblyopia impedes binocular function in the adult depends on the resolution of this issue. The most well accepted idea comes from the original animal model of Wiesel and Hubel. ${ }^{1}$ These and subsequent models argue that amblyopia is a consequence of the breakdown in binocular function caused originally by a strabismus/ anisometropia. The animal model developed by Ikeda and colleagues ${ }^{2,3}$ argues for a different relationship, one in which the binocular deficit is a consequence of the amblyopia.

Unfortunately in humans we do not know the exact ordering of these events. Our understanding of amblyopia on the one hand and stereopsis on the other has improved sufficiently over the last decade to, at least, address this important issue of whether amblyopia is an impediment to binocular function in the adult. In doing this, I will review our knowledge of stereoscopic processing, and set the nature of the amblyopic deficit within this framework.

Correspondence to: Professor R. F. Hess, McGill Vision Research Centre, Department of Ophthalmology, 687 Pine Avenue West, H4-14, Montreal, Quebec, Canada H3A 1 A1. Fax: +1 (514) 843-1691.

\section{TWO DIFFERENT TYPES OF STEREOPSIS}

The So-called Fourier Mechanism

The more traditional view of stereopsis is encapsulated in a number of what are currently referred to as 'binocular energy models'. Precortical processing is modelled as sets of bandpass filters operating at different spatial scales across the same regions of the visual image. Stereoscopic neurons extract disparity from these inputs at each of a number of scales by virtue of either a positonal-shift ${ }^{4}$ or spatial phase differences ${ }^{5}$ in their inputs. Binocular neurons which receive inputs from high-frequency cells encode small disparities while binocular neurons receiving input from low-frequency cells encode larger
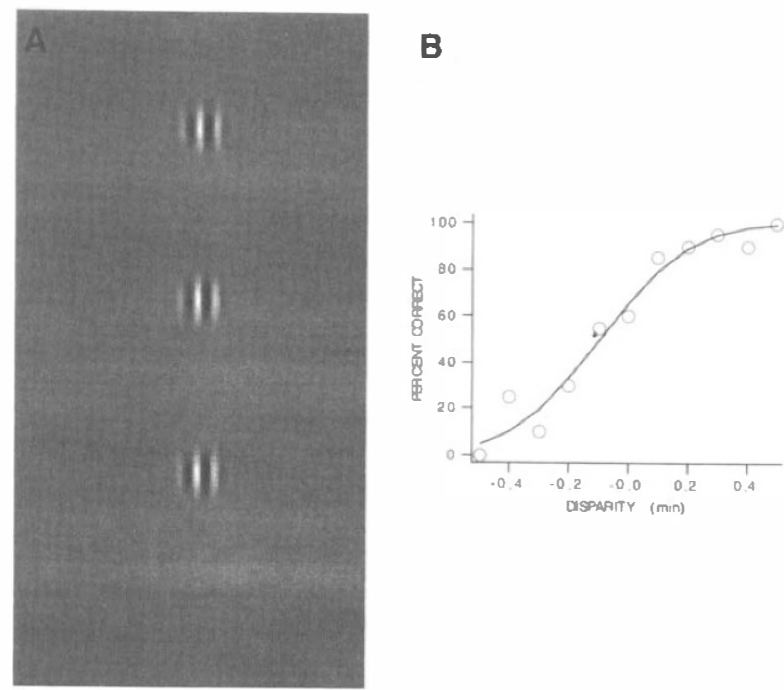

Fig. 1. (A) A three-element stimulus used to measure stereoacuity. The outer two elements are reference stimuli located in the plane of fixation while the middle element is displayed in front or behind this plane. The stimulus elements are spatially narrowband, being composed of a one-dimensional sinewave carrier multiplied by a twodimensional Gaussian envelope. (B) A typical psychometric function obtained with such a stimulus. The curve is the best fitting error function. Its standard deviation, which relates to the slope of the curve, gives an estimate of stereoacuity. 


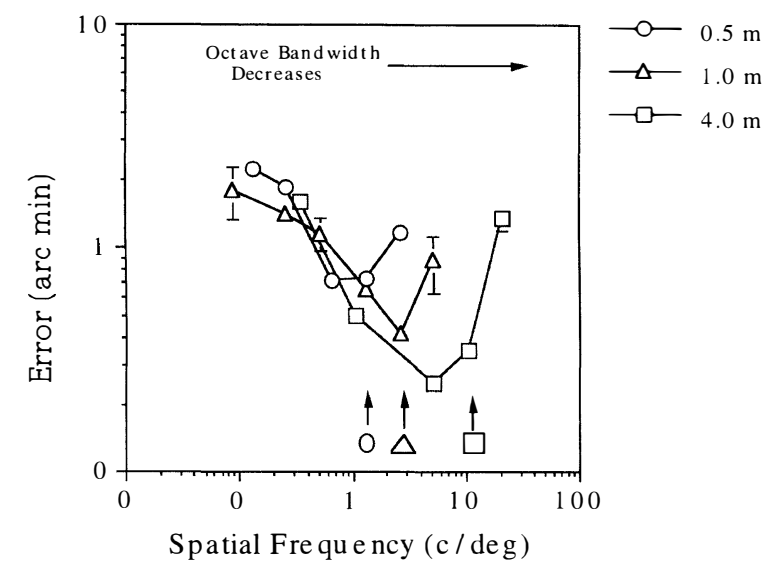

Fig. 2. The dependence of stereoacuity on the spatial frequency of the carrier for the stimulus depicted in Fig. 1 A. Results are shown for two subjects and three different envelope sizes. (From Hess and Wilcox. ${ }^{9}$ )

disparities. This is sometimes referred to as a linear/ energy model because the stereoscopic information is explicitly represented in the power spectrum.

For example, Fig. 1A shows a stimulus which we have used to measure stereosensitivity over the last few years. It comprises two outer reference elements which are located in the fixation plane and a middle element which can be displayed either in front of or behind the fixation plane. The subject's task on any one presentation is to identify whether the middle element is behind or in front of the reference elements in the fixation plane. The elements themselves are called 'Gabor elements' and they model the receptive field arrangement of the precortical filters. They are constructed by multiplying a one-dimensional sinusoid by a two-dimensional Gaussian envelope. A typical result from 220 trials is shown in Fig. 1B. The per cent in front is plotted against the grid of depth values, with zero representing the fixation plane. The error curve which is fitted to the data allows us to derive the accuracy in terms of the standard deviation parameter (equivalent to the slope of the function). The resultant accuracy represents the stereoacuity for cells with a spatial scale that matches the stimulus elements.

In Fig. 2, stereoacuity is plotted against the spatial frequency of the stimulus elements for three different sized stimuli, obtained by changing viewing distance. The lower the frequency, the poorer the stereoacuity. There is little improvement above about 2-4 cycles/ degree regardless of the overall size of the stimuli. Fig. 3 shows the dependence of disparity threshold on the interocular contrast ratio for a number of different spatial frequencies from the results of Legge and $\mathrm{Gu}^{6}$ As the ratio departs from unity, stereo performance is degraded. Similar results were found by Halpern and Blake $^{7}$ who additionally showed that at higher spatial frequencies $(5 \mathrm{c} / \mathrm{deg})$

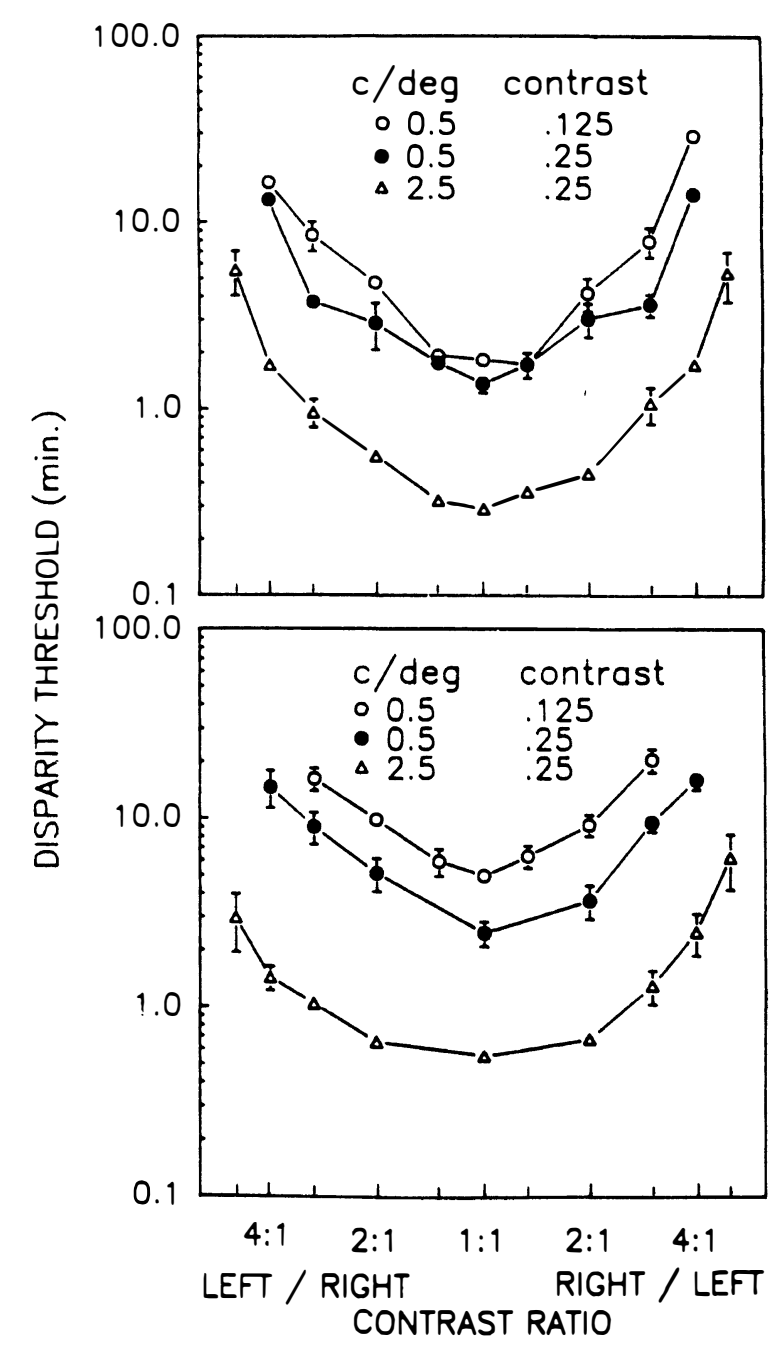

Fig. 3. The dependence of stereoacuity on the interocular contrast for extended grating stimuli. Results are shown for three different spatial frequencies. (From Legge and $\mathrm{Gu} .^{6}$ )

there was a much shallower dependence. We will see later that these two factors, namely the spatial frequency and contrast dependence, are relevant to our understanding of the impaired binocular performance of amblyopes. However, recent results suggest that there may be more to stereopsis than this socalled Fourier mechanism.

\section{The Non-Fourier Mechanism}

For some time we have been aware that motion perception can be served either by linear mechanisms which encode the oriented information in the spatial frequency versus temporal frequency domain or by non-linear mechanisms. ${ }^{8}$ Similar evidence is now available for stereopsis. ${ }^{9-14}$ For example, take the stimulus depicted in Fig. 1A. Linear stereo or motion mechanisms are interested in the periodicity of the carrier frequency not the size of the Gaussian envelope which defines the overall stimulus. Because the carrier frequency is modulated equally above and below the mean level, the information concerning the 


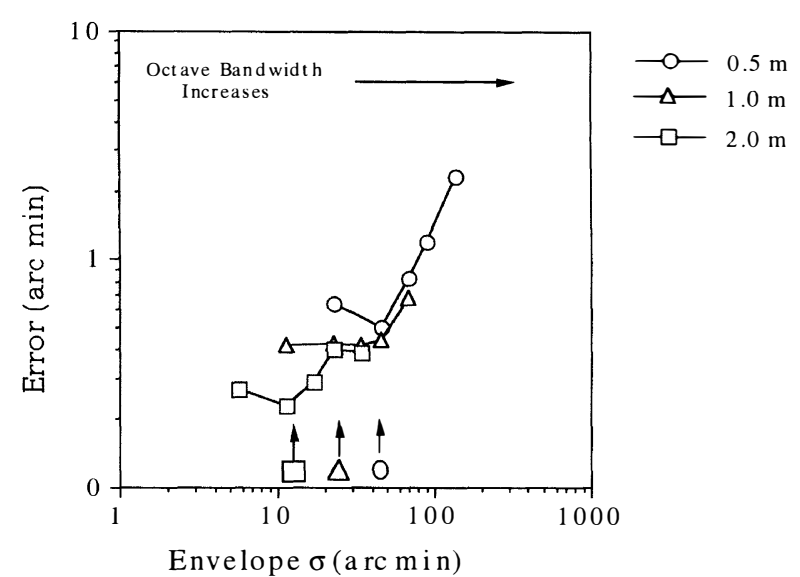

Fig. 4. The dependence of stereoacuity on the size of the two-dimensional Gaussian envelope for the stimuli of Fig. 1A. Results are shown for three different spatial frequencies of the carrier. Note that in the region of the envelope dependence there is very little dependence of carrier spatial frequency. (From Hess and Wilcox. ${ }^{9}$ )

size of the Gaussian envelope is not explicitly represented in the output of linear filters. It is represented in how the activity of the population is modulated across space rather than the firing pattern of any one cell. To make this information explicit one needs to introduce a non-linearity such as a rectification or squaring so that there will then be energy at the envelope frequency and hence in the outputs of single cells. It has been shown ${ }^{9-12}$ that stereoscopic performance depends on the size of the Gaussian envelope as well as the periodicity of the carrier. Other equally compelling demonstrations exist for non-linear processing in stereo. ${ }^{13,14}$ Hence there is evidence for a second type of stereoscopic mechanism. It is by its very nature a coarser stereo mechanism which makes a dominant contribution to our perception of large disparities rather than small disparities. ${ }^{11}$

The spatial frequency dependence of this mechanism can be gauged from the results displayed in Fig. 4 where stereo acuity is plotted against the size of the Gaussian envelope for three different carrier frequencies. In the region where there is a clear envelope dependence, there is very little dependence on the spatial frequency of the stimulus. The interocular contrast dependence of this mechanism is not known. Whether these represent two independent stereo mechanisms or just two modes of operation of a single integrative mechanism is unknown.

\section{THE NATURE OF THE AMBLYOPIC DEFICIT AND ITS RELATION TO STEREO CODING}

The amblyopic deficit consists of three main parts: acuity loss, contrast sensitivity loss and spatial uncertainty. How might each of these restrict stereo performance?

\section{Acuity Loss}

It is true that for the linear stereo mechanism, higher frequency precortical filters contribute to the processing of finer disparities. Loss of spatial resolution must therefore result in the loss of stereo acuity. However, this is unlikely to be a dominant effect in amblyopia for broad-band images. Firstly, the grating acuity loss in amblyopia is often not very severe, amblyopic resolution being often above $2 \mathrm{c} / \mathrm{deg}$. Secondly, from Fig. 2 it is seen that stereo acuity improves with increasing spatial frequency but reaches a plateau at around $2-4 \mathrm{c} / \mathrm{deg}$. It is only for those amblyopes whose spatial acuities are significantly below $2 \mathrm{c} / \mathrm{deg}$ that this would be a significant factor. The resolution loss would probably not affect performance for the non-linear stereo mechanism at all.

\section{Contrast Sensitivity Loss}

Possibly of more importance than the restricted spatial range is the loss of contrast sensitivity within the passband of the amblyopic visual system. While stereo acuity is reasonably insensitive to the absolute contrast level, it is sensitive to interocular differences in contrast. ${ }^{6,7}$ This is the relevant factor for amblyopia. For example, both studies found an approximately proportional change in stereo acuity with interocular contrast for low-medium spatial frequencies (1.2-2.4 c/deg). However, at higher spatial frequencies (around $4.8 \mathrm{c} / \mathrm{deg}$ ) the effect of interocular contrast differences is small. Since stereo acuity is insensitive to interocular contrast changes above 2 $\mathrm{c} / \mathrm{deg}$ one would not expect contrast sensitivity losses in amblyopia, which are usually significant in this range, ${ }^{15,16}$ to impair stereoscopic function greatly. This is particularly true since reduced threshold contrast sensitivity and perceived suprathreshold contrast are disassociated in strabismic and to a lesser extent anisometropic amblyopia. ${ }^{17}$ Stimuli for which amblyopes exhibit dramatic elevations in threshold are not perceived as being reduced when they are above threshold.

In summary, in the majority of amblyopes whose contrast sensitivity is significantly reduced but whose grating acuity is above 2-4 c/deg, one would not expect, on the basis of what we presently know about normal stereopsis, their stereo performance to be greatly compromised by their spatial filtering deficit per se. This conclusion assumes that the mechanisms responsible for stereopsis and dichoptic contrast masking ${ }^{18}$ (discussed by Harrad in this issue) are different. Very severe deficits to contrast sensitivity will affect linear more than non-linear stereo 
computations because of the former's stronger spatial frequency dependence.

\section{Positional Uncertainty}

Amblyopes exhibit positional uncertainty for stimuli both at ${ }^{19}$ and below ${ }^{20}$ the acuity limit. The positional loss exhibited by anisometropic amblyopes can be explained on the basis of their known acuit ${ }^{21}$ and contrast sensitivity ${ }^{20}$ losses. On the basis of this, one would not expect anisometropia (except in its most severe form) to be an impediment to binocular function. There is evidence that for anisometropic amblyopes, stereo performance is normal for equivisible low spatial frequency stimuli ${ }^{22}$ but not for high spatial frequency stimuli. These results are shown in Fig. 5. On the basis of this one has to conclude that the loss of binocular function in the high frequency range has a binocular rather than a monocular cause.

The positional loss in strabismic amblyopes is large even when acuity and contrast sensitivity losses are taken into account. ${ }^{19,20}$ Our working model ${ }^{23}$ involves a disruption of the local sign of detectors due to a topological distortion of the spatial map in the cortex fed by the amblyopic eye. It is not just that the cortical inputs are miswired, as some have argued (T. Rentschler, personal communication) but that the neurons in V1 are disarrayed. Furthermore, it seems

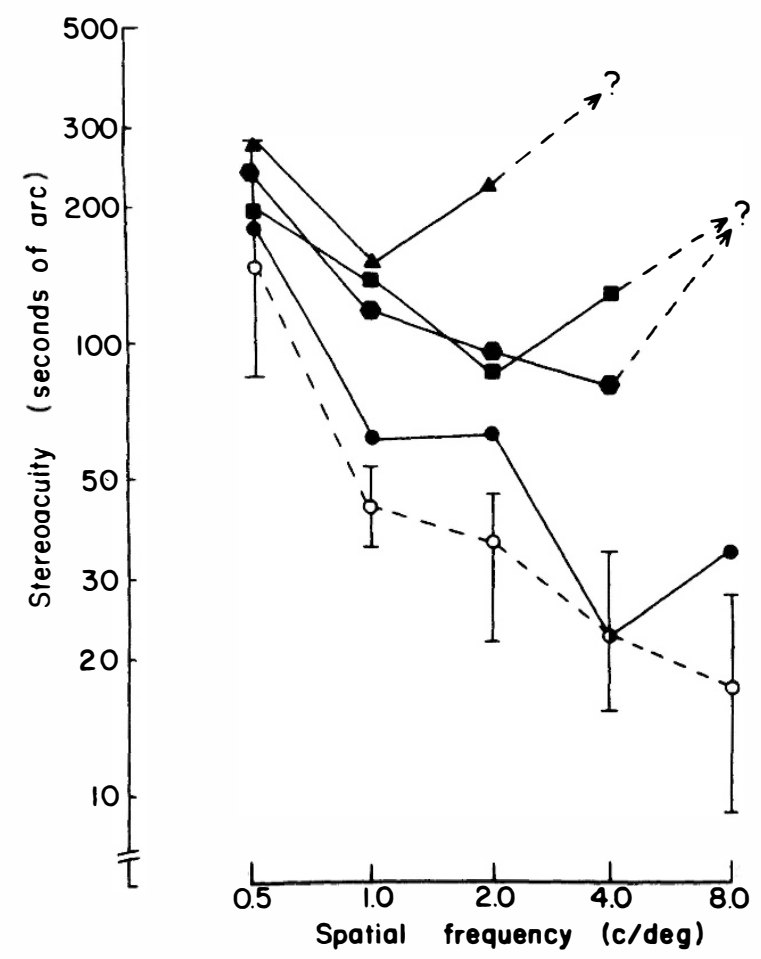

Fig. 5. Dependence of stereoacuity on spatial frequency of windowed gratings for normals (open symbols) and a group of four anisometropic amblyopes (filled symbols). The stimuli were matched for perceived contrast between the normal and fellow amblyopic eyes. Notice that stereoacuity is normal at low spatial frequencies but abnormal at high spatial frequencies. (From Holopigian and Blake. ${ }^{22}$ )

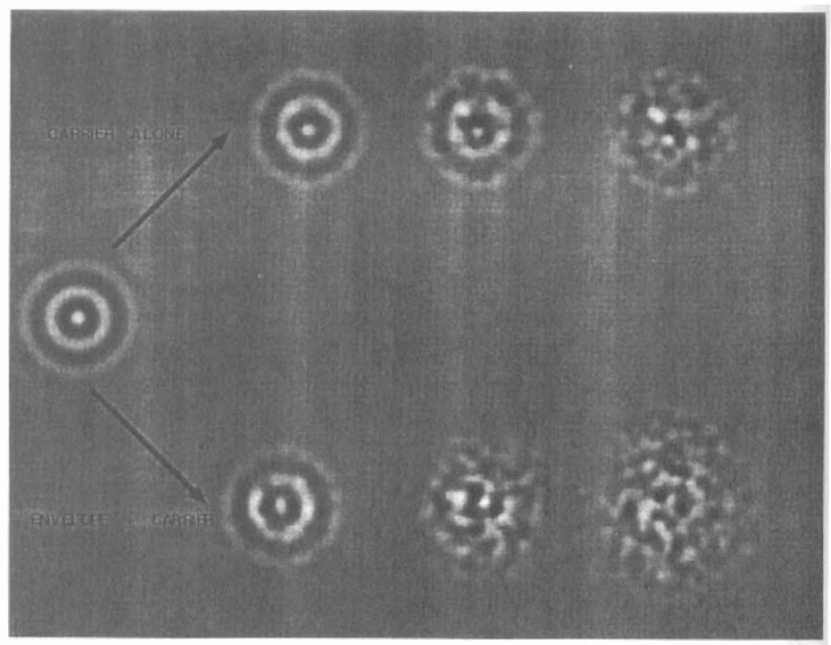

Fig. 6. Example of radial sinusoidal stimuli which have been jumbled through an array of matched bandpass filters. At the top, just the carrier is jumbled. At the bottom, both carrier and envelope are jumbled.

likely that this disruption involves low as well as high spatial frequency neurons. ${ }^{20}$ This is potentially a much more serious problem for stereopsis than either the resolution or contrast losses described above. The strength of the stereo signal after the information from the two eyes has been combined is given by the degree to which the two signals are correlated. $^{24}$ If one eye contributes a jumbled percept which is scale invariant then the interocular correlation will be low and the binocular signal weak. Furthermore, since the jumbling is not confined to the inputs of cortical cells but affects their outputs as well, it will affect both linear and non-linear stereo processing streams. An illustration, of two different types of receptive field jumbling is shown in Fig. 6.

At the top of Fig. 6 the carrier frequency is

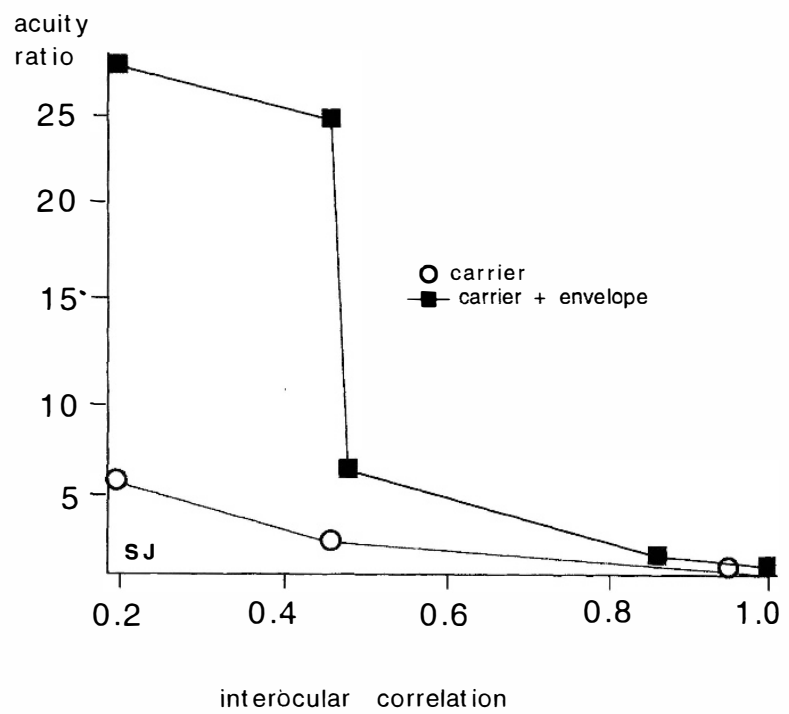

Fig. 7. The dependence of stereoacuity on interocular correlation for stimuli jumbled as in Fig. 6. For the same degree of interocular correlation, disrupting the carrier and the envelope affects stereo performance more than just disrupting the carrier alone. (From Jovkar et al. ${ }^{25}$ ) 
jumbled by an array of filters tuned to its periodicity. At the bottom of the figure, the stimulus as a whole (carrier + envelope) is jumbled by a matched receptive field array. The process of creating these examples of what might be the basis of the positional disturbance in amblyopia involves a sampled convolution with an array of matched filters with a disorder incorporated into the reconstruction process. The power spectra of the resultant stimuli are little changed; however, the phase spectrum is severely disrupted. From what we know of the severity of the positional deficit in amblyopia, the better model is captured by the distortions at the bottom of Fig. 6 .

The graph in Fig. 7 shows the relationship between the degree of interocular correlation, our measure of stereo strength, and stereoacuity. ${ }^{25}$ This experiment was carried out at only one scale (frequency of the radial sinusoid) but the results are applicable to any scale assuming that the filters are jumbled by some fraction of their size. ${ }^{20}$ Stereoacuity is affected. In the case where the distortions are applied to the envelope as well as the carrier (Fig. 6, bottom, represents the stimulus), which is the more realistic case on the basis of what we know about amblyopia, stereoacuity is much more dramatically affected. Such a manipulation would be expected to affect both linear and non-linear stereo processing.

In conclusion, the above analysis suggests that in anisometropic amblyopia only the more severe contrast sensitivity losses will limit stereo performance. The observed deficit ${ }^{22}$ in stereo performance in these amblyopes represents binocular dysfunction unimpeded by the accompanying amblyopia.

In strabismic amblyopia where there are independent losses of contrast sensitivity and positional accuracy, the greatest obstacle to stereopsis is not the contrast (amplitude) losses but the position (phase) losses. These are best thought of in terms of a spatial disarray of filters that optimally sample space and spatial frequency. These losses potentially affect all scales and set an upper limit on the degree of stereopsis that can be achieved by an amblyopic visual system, because they will not only affect the small disparities signalled by the linear system but also the coarser disparities signalled by the non-linear system.

This work was supported by the Canadian Medical Research Council (\# MT 108-18). I am very grateful to my colleagues Laurie Wilcox, David Simmons and Said Jovkar for allowing me to use some of our recent material (Fig. 7).

Key words: Amblyopia, Contrast sensitivity, Spatial frequency, Stereopsis.

\section{REFERENCES}

1. Wiesel TN, Hubel DH. Comparison of the effects of unilateral and bilateral eye closure on cortical unit responses in kittens. J Neurophysiol 1965;28:1028-40.
2. Ikeda H, Wright MJ. Properties of LGN cells in kittens raised with convergent squint: a neurophysiological demonstration of amblyopia. Exp Brain Res 1976; 25:63-77.

3. Ikeda H, Tremain KE. Amblyopia occurs in retinal ganglion cells in cats reared with convergent squint without alternating fixation. Exp Brain Res 1979;35: 559-82.

4. Barlow HB, Blakemore C, Pettigrew JD. The neural mechanism of binocular depth discrimination. J Physiol (Lond) 1967;193:327-42.

5. DeAngelis GC, Ohzawa U, Freeman RD. Depth is encoded in the visual cortex by a specialised receptive field structure. Nature 1991;352:156-9.

6. Legge GE, Gu Y. Stereopsis and contrast. Vision Res 1989;29:989-1004.

7. Halpern DL, Blake RR. How contrast affects stereoacuity. Perception 1988;17:483-95.

8. Chubb C, Sperling, G. Drift-balanced random stimuli: a general basis for studying non-Fourier motion perception. J Opt Soc Am 1988;5:1966-2007.

9. Hess RF, Wilcox LM. Linear and non-linear filtering in stereopsis. Vision Res 1994;34:2431-8.

10. Wilcox LM, Hess RF. Linear and non-linear contributions to stereopsis. Invest Ophthalmol Vis Sci (Suppl) 1993;34:2382.

11. Wilcox LM, Hess RF. $D_{\max }$ for stereopsis depends on size not spatial frequency content. Vision Res 1995;35:1061-9.

12. Wilcox LM, Hess RF. Is the site of non-linear filtering in stereopsis before or after binocular combination? Vision Res 1994;34:2431-8.

13. Sato T, Nishida S. Second-order depth stimuli with texture-defined random check stereograms. Invest Ophthalmol Vis Sci (Suppl) 1993;34:1438.

14. Sato T, Nishida S. Does an envelope detecting mechanism mediate stereopsis for band-limited stimuli? Invest Ophthalmol Vis Sci (Suppl) 1994;35:1916.

15. Hess RF, Howell ER. The threshold contrast sensitivity deficit in strabismic amblyopia: evidence for a two type classification. Vision Res 1977;17:1049-55.

16. Levi DM, Harwerth RS. Spatio-temporal interactions in anisometropic and strabismic amblyopia. Invest Ophthalmol Vis Sci 1977;16:90-5.

17. Hess RF, Bradley A. Contrast perception above threshold is only minimally impaired in human amblyopia. Nature (Lond) 1980;287:463-4.

18. Harrad RA, Hess RF. Binocular integration of contrast information in amblyopia. Vision Res 1992;32:2135-50.

19. Levi DM, Klein SA. Differences in acuity for gratings between anisometropic and strabismic amblyopes. Invest Ophthalmol Vis Sci 1982;23:398-407.

20. Hess RF, Holliday IE. The spatial localization deficit in amblyopia. Vision Res 1992;32:1319-39.

21. Levi DM, Klein SA. Vernier acuity, crowding and amblyopia. Vision Res 1985;25:979-91.

22. Holopigian K, Blake RR. Selective losses in binocular vision in anisometropic amblyopes. Vision Res 1986; 26:621-30.

23. Hess RF, Field DJ. Is the spatial deficit in amblyopia due to loss of cells or disarray of cells? Vision Res 1994;34:3397-406.

24. Cormack LK $\dot{K}$, Stevenson SB, Schor CM. Interocular correlation, luminance contrast and cyclopean processing. Vision Res 1991;31:2195-207.

25. Jovkar S, Hess RF, Wilcox LM, Simmons D. Effect of image distortions on stereoscopic performance. Invest Ophthalmol Vis Sci (Suppl) 1995;36:366. 